\title{
Eugenesia. Un análisis histórico y una posible propuesta
}

\author{
Fabiola Villela Cortés ${ }^{1}$, Jorge E. Linares Salgado²
}

Resumen: La eugenesia es un tema abordado, entre otros, por historiadores, filósofos, médicos, bioeticistas, por distintas razones. La idea de mejorar la raza humana siempre ha estado presente en la historia de la civilización, también han sido objeto de comentarios el intento de consolidarla como una ciencia, la presencia de movimientos eugenésicos en varios países del mundo, el holocausto nazi y, finalmente, el resurgimiento de la eugenesia a raíz de la decodificación del genoma humano. Nuestro objetivo es dar un repaso por los movimientos eugenésicos que tuvieron lugar a mediados del siglo XX, el resurgimiento de la eugenesia y los adelantos con los que contamos actualmente.

Palabras clave: eugenesia totalitaria, eugenesia liberal, genética

\section{Eugenics. A historical analysis and a possible proposal}

\begin{abstract}
Eugenics is a topic treated for several reasons by, among others, historians, philosophers, physicians, bioethicists. The idea to improve human race always has been present in the history of civilization, to try to consolidate it as a science also has been the object of commentaries, the presence of eugenic organizations in several countries, the nazi holocaust and finally, the eugenic resurgence since the human genome sequencing. Our objective is to review eugenic movements in the mid twentieth century, eugenic resurgence and current advancements.
\end{abstract}

Key words: totalitarian eugenics, liberal eugenics, genetics

\section{Eugenia. Uma análise histórica e uma possível proposta}

Resumo: A eugenia é um tema abordado, entre outros, por historiadores, filósofos, médicos, bioeticistas, e por diferentes razóes. A ideia de melhorar a raça humana sempre tem estado presente na história da civilização. Também tem sido objeto de comentários a intenção de consolidá-la como uma ciência a presença de movimentos eugênicos em vários países do mundo, o holocausto nazista e, finalmente, o ressurgimento da eugenia na raíz da decodificaçáo do genoma humano. Nosso objetivo é dar um repasso pelos movimentos eugênicos que tiveram lugar em meados do século XX, o ressurgimento da eugenia e os avanços com os quais contamos atualmente.

Palavras-chave: eugenia totalitária, eugenia liberal, genética

\footnotetext{
${ }^{1}$ Secretaria Académica, Posgrado en Filosofía de la Ciencia, Universidad Nacional Autónoma de México, México

${ }^{2}$ Coordinador Posgrado en Filosofía de la Ciencia, Programa de Maestría y Doctorado en Ciencias Médicas, Odontológicas y de la Salud, Universidad Nacional Autónoma de México, México

Correspondencia: fabiola.villela@gmail.com
} 


\section{Introducción}

Al revisar la historia de la eugenesia nos damos cuenta de que la búsqueda de la perfección en el ser humano no es nueva, ya que desde la antigua Grecia se tiene registro de distintos proyectos en diferentes contextos históricos que se propusieron alcanzar esta meta. Después de estudiar varios de estos proyectos sociales podemos concluir que hay tres elementos constantes: a) la idea de que es posible perfeccionar al ser humano, b) la existencia de subhumanos, es decir seres que no son considerados como personas, y c) la idea de perfección biológica y psicológica ligada al progreso en distintos sentidos sociales.

La historia del siglo XX nos enseñó que dejar completamente en manos de un Estado totalitario los criterios y fines del perfeccionamiento humano termina con la lúgubre realidad de los campos de concentración. Esto es, que un modelo de eugenesia dirigido y controlado por el poder político conducirá muy probablemente a una catástrofe moral.

En un esfuerzo por mejorar la situación social, recuperando los fines más positivos de la eugenesia, y frente a la lucha por las libertades individuales que ha caracterizado la historia social reciente desde la segunda mitad del siglo XX, la alternativa de varios autores consiste en entregar a la autonomía individual la capacidad para dirigir los criterios de tal perfeccionamiento humano, y que además sea el mercado el que dirija y regule el desarrollo de la eugenesia. Sin embargo, cabe preguntarse si solo tenemos estas dos opciones: o la eugenesia totalitaria o la eugenesia liberal controlada por el mercado capitalista.

El objetivo de este trabajo es dar un repaso a los movimientos eugenésicos que tuvieron lugar a mediados del siglo XX y al resurgimiento de la eugenesia posterior al descubrimiento del ADN, denominada "eugenesia liberal", y delinear una propuesta de eugenesia que se encuentre entre ambos movimientos.

\section{Método}

Se realizó una revisión bibliográfica acerca de los dos movimientos eugenésicos mencionados (totalitario y liberal); posteriormente, un análisis de ambos, con el objetivo de rescatar lo mejor de cada uno para, sobre esa base, proponer los lineamientos preliminares de un tercer modelo eugenésico.

La eugenesia o "bien nacer" fue el término acuñado por el naturalista británico Francis Galton en 1883. El interés de Galton por la eugenesia surgió poco después de la publicación del "Origen de las especies" (1859), escrito por su primo Charles Darwin. Con la convicción de que el talento, la habilidad, la inteligencia y otros factores "corrían en las familias" y que la selección natural interviene en el ser humano de igual forma que en las demás especies, Galton sugirió que, así como el hombre había obtenido extraordinarias razas de caballos y perros, se podía mejorar la raza humana controlando la reproducción(1).

Galton se aprovechó del momento histórico para introducir, a inicios del siglo XX, su campaña en pro de la eugenesia como "política de Estado", mediante sociedades y grupos intelectuales influyentes(2).

Para comprobar su hipótesis, Galton estudió y describió pedigríes familiares de personas famosas (jueces, gobernadores, militares, científicos, poetas, músicos, etc.), concluyendo que hombres distinguidos provienen de familias distinguidas. Los resultados de su estudio se publicaron en "Heredetary talent and character", en la revista McMillan's Magazine, en 1865, y posteriormente extendió su trabajo y publicó el libro "Hereditary genius", en 1869.

Una vez comprobó que la herencia prevalece sobre todo lo demás, Galton se dedicó a planear la aceleración de la evolución humana hacia la perfección, mediante el uso de técnicas positivas y negativas.

La eugenesia positiva buscaba conservar las características de los mejores elementos que conformaban la parte hegemónica de la sociedad, así como prohibir el mestizaje para evitar la "degeneración" de una población que se consideraba homogénea. Estas medidas consistían en favorecer la unión entre jóvenes idóneos para la sociedad y patrocinar 
el matrimonio de la joven pareja con la esperanza de que procrearían hijos sanos, dotados de las cualidades adecuadas.

La eugenesia negativa se caracterizó por limitar los derechos reproductivos individuales en aras de la salud genética de las generaciones futuras, y consistía en la eliminación de caracteres indeseables mediante segregación sexual y racial, restricciones de inmigración (principalmente en EE.UU., México, Brasil, Alemania), prohibición legal de matrimonios "interraciales" y esterilización involuntaria. Otras medidas de eugenesia negativa fueron en un inicio el infanticidio $y$, posteriormente y hasta la segunda mitad del siglo XX, el genocidio(3). De forma generalizada, las medidas negativas de la eugenesia, como la esterilización y la segregación, estaban destinadas a las clases bajas, con el objetivo último de "desaparecerlas", mientras que las medidas positivas estaban enfocadas a mejorar las clases media y alta de las sociedades permitiendo su auge(4).

Un factor relevante es que en esa época, a diferencia de nuestros días, el personal encargado de la recolección de datos no necesitaba una especialización previa y, por tal motivo, psicólogos y psiquiatras fungieron como "genetistas", debido a su habilidad para definir fenotipos del comportamiento humano(4). La observación empírica apuntaba a que el alcoholismo, la prostitución o la locura se heredaban de la misma forma que la hemofilia, el daltonismo o la ceguera, y por tanto debían ser eliminados, pues "la política sanitaria (buscaba controlar) las patologías médicas y sociales consideradas como amenazas para el progreso" (5:5).

A finales del siglo XIX, muchos psiquiatras fueron perdiendo la esperanza de encontrar la cura de diferentes trastornos mentales, pues se convencieron que dichos padecimientos eran estrictamente hereditarios. Gracias a los movimientos eugenésicos, el miedo a la decadencia biológica de la especie provocó que los pocos esfuerzos enfocados a la obtención de nuevos tratamientos para pacientes psiquiátricos pasaran a segundo plano $\mathrm{y}$, en su lugar, la atención se centró en los problemas que provocaban estos pacientes a la sociedad, así como los costos elevados de mantenerlos institucionalizados. Era obvio que la mejor opción para la sociedad era que este tipo de individuos no se reprodujeran y, mejor aún, que no nacieran(6). Fue así como pasaron de la eugenesia a la "eutanasia”.

En el ambiente político de la primera mitad del siglo XX había una cierta aceptación social de esas medidas eugenésicas. Esta mezcla entre genética y sociedad se denominó "darwinismo social"3 $(1,2)$.

Uno de los cambios más importantes de esta época fue el redescubrimiento de los experimentos del fraile austríaco Gregor Mendel, ya que las "leyes de la segregación" permitieron dejar de lado la especulación, descripción y estudios cualitativos que se realizaban en ese momento con fines eugenésicos, para dar paso al estudio experimental de la evolución. Queda claro que no todos consideraban los descubrimientos de Mendel plausibles; sin embargo, gracias a los estudios realizados por Bateson en Gran Bretaña y Thomas Hunt Mor$\operatorname{gan}^{4}$ en EE.UU., fue que las leyes de segregación entraron en el mundo eugenésico(4).

En todos los países en los que durante el siglo XX se llevó a cabo un movimiento eugenésico apoyado por el gobierno, se desarrollaba un proceso histórico común: acelerada industrialización, urbanización e inmigración, tres características que provocaron la inconformidad de las clases medias, pues veían en los inmigrantes extranjeros y los campesinos pobres que migraban también a las ciudades una amenaza para el orden social. Por tanto, al encontrar en la eugenesia una alternativa científica y biológica para resolver estos problemas, dieron su apoyo a los pequeños grupos de élite que introdujeron y lideraron, sobre todo al inicio, los movimientos eugenésicos en sus países.

La idea de eugenesia que predomina en este periodo es la denominada totalitaria; esto es, realizada por Estados con gobiernos que ejercieron un poder centralizado y autoritario. El movimiento

\footnotetext{
${ }^{3}$ Teoría filosófica y sociológica que emergió durante la segunda mitad del siglo XIX y fue elemento básico del movimiento eugenésico en Inglaterra. Realizaba analogías entre el darwinismo (teoría de la evolución) y el desarrollo socioeconómico, argumentando que los individuos, grupos y sociedades en general, están sujetos a las mismas leyes de la selección natural. El proceso de selección natural llevaría a la mejora de la raza humana y la supervivencia de los mejores sistemas políticos, económicos y sociales.

${ }^{4}$ Premio Nobel de medicina en 1933, por su trabajo en genética con moscas de la fruta.
} 
eugenésico de la Alemania nazi fue el más poderoso y más cruel de todos. Pero también naciones liberales y formalmente democráticas, como EE.UU. e Inglaterra, buscaban preservar su plasma germinal, por lo cual las libertades individuales pasaron a segundo plano y la consistencia racial de la sociedad se convirtió en un objetivo importante para las políticas de Estado. Pero esta modalidad de eugenesia de Estado sucedió de forma diferente en países latinoamericanos, donde el mestizaje, característica singular de países como el nuestro, era considerado como el mal principal y, por tanto, el objetivo eugenésico fundamental en estos países era "blanquear" la raza para mejorar su plasma mestizo. Los siguientes apartados describen brevemente cómo fueron los movimientos eugenésicos en EE.UU. e Inglaterra, dos países que tuvieron en común los conocimientos científicos y de herencia que caracterizarían sus movimientos eugenésicos; posteriormente, los casos de Brasil y de México, dos países típicamente mestizos en los que el neolamarkismo y el darwinismo social marcaron la línea que habrían de seguir respecto a la eugenesia, y finalmente el movimiento nazi que sumaría todas las características mencionadas.

\section{EE.UU. e Inglaterra}

Durante la primera mitad del siglo XX, el movimiento eugenésico, tanto en EE.UU. como en Inglaterra, unió a radicales y conservadores por igual. Sin embargo, los más entusiastas en ambos países pertenecían principalmente a la clase media o media alta, eran anglosajones (aunque en Inglaterra había miembros de la comunidad judía), mayoritariamente protestantes y con educación escolar alta. De tal forma, el movimiento eugenésico permitió a la clase media forjarse un lugar de poder social, que tuvo lugar como efecto de la industrialización y la migración de las provincias hacia las grandes ciudades.

Muchos eugenistas esperaban que sus programas de mejoramiento de la raza, positivos o negativos, fueran voluntarios, de ahí el énfasis en la educación, el mandato moral y la necesidad de aplicar la anticoncepción. El control sobre las áreas más privadas de la vida, como el matrimonio, el sexo y el embarazo, se dejaron en un principio en lo privado(4:90). Pero el gobierno norteamericano no tardó en prever que debía hacer algo más para evitar el deterioro del plasma poblacional, por lo que un mecanismo para evitar esto fue institucionalizar el matrimonio.

Ejemplo ilustrativo fue que el Estado de Indiana implementó una medida tripartita, aprobada en 1905, que 1) prohibía el matrimonio de deficientes mentales y bebedores habituales, 2) requería un certificado de salud de todas las personas liberadas de instituciones mentales y 3) declaraba inválidos los matrimonios que esas personas, para evitar la ley estatal, habían contraído en otro Estado(4:99-100). Poco a poco, otros Estados siguieron el ejemplo de Indiana, pues las leyes de migración de este país fueron emitidas en el nivel federal y esta fue una de las grandes diferencias entre EE.UU. e Inglaterra con relación a la promulgación de leyes eugenésicas.

Desde 1875, EE.UU. había comenzado a poner trabas a la inmigración, aunque en aquel entonces estas solo alcanzaban a extranjeros "indeseables”, entre los que se encontraban prostitutas y ex convictos. Con el transcurso de los años fueron añadiéndose gradualmente otros grupos: en 1882 "lunáticos e idiotas", en 1903 "epilépticos e insanos", en 1907 “imbéciles y débiles mentales". Hasta que se aprobó la Ley de inmigración Jonson-Logge, de 1924, que se proponía estimular el proceso de purificación racial(2:121).

En EE.UU. la mayor cantidad de migrantes eran refugiados económicos, pobres, analfabetos y con problemas de salud. Mientras algunos reformistas se preocupaban por mejorar la calidad de vida de los pobres, otros pensaban que la falta de oportunidades, los bajos sueldos, la vivienda inadecuada y los problemas sanitarios que conducían a la mayoría de las poblaciones marginadas —entre ellos los migrantes - a la pobreza, la enfermedad y los actos criminales, no eran un problema social, sino de herencia genética y, por tanto, si se podía evitar que estas personas se reprodujeran, entonces el crimen, la pobreza y la enfermedad se eliminarían(5-7).

La razón por la cual la esterilización se convirtió en el elemento clave para el movimiento eugenésico de todos los países estudiados fue que era 
concebida como un método preventivo enfocado a proteger a la sociedad de los retrasados mentales, los criminales, los idiotas, etc.

El desplazamiento de la eugenesia de Inglaterra a Estados Unidos favoreció la temprana incursión de la eugenesia en el resto de América y el papel protagónico que adquirió la propuesta norteamericana en la conformación de un proyecto panamericano sería evidente en los próximos años(5:6).

\section{Brasil y México}

Puesto que la biología evolutiva y la genética eran ciencias muy recientes, el intento de circunscribir la eugenesia a estos dos campos fracasó, sobre todo en países latinoamericanos, en los cuales no se estaban realizando estudios científicos. En su lugar, la puericultura (cuidado materno infantil) y la homicultura (cuidado de la vida de los sujetos) se convirtió en parte medular de los movimientos eugenésicos del mundo iberoamericano, en los que se consideraban problemas socioeconómicos y medioambientales como parte de la eugenesia.

\section{Brasil}

El primer país en Latinoamérica en tener un movimiento eugenésico significativo fue Brasil. Este se inició en 1917 y concluyó en 1940; sin embargo, previamente comenzaron a darse las condiciones sociales para el auge de la eugenesia. De 1900 a 1940 el país sufrió cambios sociales y políticos profundos debido a una industrialización tardía, urbanización y gran inmigración europea, aunadas a una emancipación de la esclavitud bastante reciente (1888) y un sentimiento nacionalista creciente.

Brasil representaba para los europeos y la elite del país el epítome de la disgenesia, pues era una nación pobre, subdesarrollada, con una población mayoritariamente rural, iletrada, católica y racialmente mezclada. Los científicos brasileños pensaban que el desarrollo científico debía seguir los pasos del primer mundo, ya que veían en la ciencia un signo de modernidad cultural. Hasta 1920 casi no había centros de investigación enfocados al estudio de la biología darwiniana y la genética, convirtiendo a Brasil en un país más consumidor que productor en el área científica(8).

Por tanto, la gran aceptación del movimiento eugenésico se debió a la promesa de un "nuevo orden social", visto por la clase alta brasileña como el poder de la ciencia que daría "orden y progreso" 5 al país. No obstante, la noción de eugenesia que se desarrolló fue diferente a la norteamericana o inglesa.

La concepción de eugenesia que permaneció y se difundió en América Latina no se basaba en concepciones mendelianas, sino en neolamarkianas ${ }^{6}$, provenientes de Francia, en las que no había una diferencia marcada entre "naturaleza" y "crianza" que permitiera mejorar la herencia en un solo sentido.

Por tanto, para muchos científicos, políticos y médicos, el movimiento eugenésico era visto como una nueva rama de la higiene ${ }^{7}$ y fue lo que permitió a los brasileños reclamar "sanear é eugenizar" (sanear es eugenizar). Las medidas eugenésicas implementadas se pueden dividir en dos: campańas de salud pública, enfocadas a prevenir enfermedades venéreas, alcoholismo, campañas cuidado prenatal, ejercicio, etc., y, por otro lado, las propiamente eugenésicas, como el aborto o la contracepción(9).

\section{México}

El momento en el cual nace el movimiento eugenésico en México es fundamental para comprender su desarrollo. La eugenesia llegó a este país al mismo tiempo que se llevaba a cabo la última fase de la Revolución Mexicana y esperó el momento preciso para insertarse en la reconstrucción del país y la institucionalización del Estado Revolucionario, en el que la pregunta ¿cuáles deberían ser las características del nuevo pueblo nacional? le abrió las puertas de esta nación(10).

\footnotetext{
${ }^{5}$ Lema republicano que ondea en la bandera brasileña.

${ }^{6}$ Una teoría basada en los preceptos lamarkianos de caracteres adquiridos que pasan de generación en generación debido a la ventaja que ofrece para la especie.

${ }_{7}$ Olegario de Moura, presidente de la Sociedad Eugenésica de Sao Paulo, sostenía que el saneamiento era lo mismo que la eugenesia, solo que el primer término era más popular, mientras el segundo más científico (de Moura, 1919b, p. 83; en Leyes, N., 121; en Adams, M., 1990).
} 
La situación social en el país se podía definir como guerra racial, debido a la heterogeneidad étnica. La concepción de "pueblo mexicano" después de la Revolución se mezcló con el darwinismo social. Dejando atrás el Porfiriato, el optimismo posrevolucionario acudió a las disciplinas sociales para definir los principios de la "nación", retomando el glorioso pasado indígena y el potencial educativo del pueblo mexicano(5:11).

La eugenesia mexicana cubría tres grandes vertientes: 1) el racismo, centrado en problemas de inmigración, 2) la puericultura que, como mencionamos, es básicamente una propuesta educativa y de protección al cuidado materno-infantil, y 3) la profilaxis médico-sanitaria, interesada en prevenir las patologías clínicas y sociales a partir de un diagnóstico temprano $(5: 7,10)$.

Se buscaba "blanquear" la población, de preferencia con sangre europea, y mejorar así la raza. Al igual que en otros países, se mezclaron medidas propiamente eugenésicas con medidas de prevención en la salud.

Como en otros países, la protección que se dio a las mujeres fue ambigua. Por un lado, se favoreció la salud materno-infantil, la educación sexual, la maternidad responsable, la anticoncepción y el aborto, pero, por otra parte, se difundió que el ámbito natural de las mujeres era la familia y su principal función la procreación(11:310).

\section{Alemania nazi}

Para los nazis, la eugenesia se convirtió en la base de su Reich de mil años y la búsqueda de la perfección radicaba en la purificación de la raza aria. En el centro de la ideología nazi se encontraba la idea de que la sociedad era un organismo vivo y la sociedad alemana formaba una "sociedad de sangre", de ahí que los individuos no fueran tan importantes como la sociedad. Los intereses de la sociedad se encontraban sobre los de los individuos y era la sociedad la que debía prevalecer. La funcionalidad o disfuncionalidad de los individuos afectaba la salud de ese organismo social, de ahí que la higiene racial se volviera tan importante para esta ideología(12).
Desde el principio, el gobierno nazi vio la herencia humana como un recurso científico que podía promover y legitimar las políticas raciales. Por eso el régimen proveyó apoyo financiero a instituciones como el Instituto de Antropología, Herencia Humana y Eugenesia Kaiser Wilhelm (KWIA) (13).

Pasó poco tiempo para que el gobierno llegara a la conclusión de que los "indeseables", esto es, insanos mentales, discapacitados y enfermos, no debían nacer. Evitar su reproducción era igualmente importante, ya que los análisis poblacionales indicaban que los inferiores se reproducían más y más rápido. Tanto científicos como políticos orgullosamente nacionalistas, preocupados por el deterioro de su raza, idearon diferentes mecanismos para evitar el declive. Por ejemplo, en 1933 se aprobó la ley que permitía la esterilización forzada de enfermos mentales. Dos años después, en 1935, fueron aprobadas las leyes de Nürenberg, enfocadas en "ariar" la sangre alemana, redefinir la ciudadanía y evitar el matrimonio o cualquier contacto sexual entre judíos y alemanes $(3,14)$.

Pero no todas las acciones eugenésicas fueron legales. En 1937 se esterilizó ilegalmente a cientos de niños y comenzó el programa para esterilizar a más de treinta mil gitanos alemanes. Para 1940, 70 mil pacientes psiquiátricos habían sido eliminados en las cámaras de gas y en 1941 entraba en vigor la esterilización masiva de judíos puros y mestizos(3).

La Ley de Esterilización Eugenésica de 1933 fue solo el primer paso. Para mejorar la raza se daban incentivos económicos. Heinrich Himmler impulsó a miembros de la S.S. a procrear muchos niños con mujeres racialmente adecuadas y en 1936 institucionalizó el Lebensborn (hogares tipo spa donde las mujeres, casadas o no, tenían acceso al mejor cuidado médico durante su confinamiento) (4:117). Se realizaron campańas supervisadas por el ministro de propaganda, Joseph Goebels, el cual declaró que todas las facetas de la vida alemana debían ser informadas por una forma eugénesica de pensar. Los doctores y las parteras, o matronas, se convirtieron en los guardianes de la salud y, por tanto, de la nación(14). Alemania dividió su atención racial en tres vías: 1) cuidado médico para el individuo, 2) salud pública para la 
comunidad y 3) eugenesia para la raza(15).

Al concluir la Segunda Guerra Mundial el mundo se percató de las atrocidades cometidas en Alemania y la eugenesia se convirtió en tabú. Para 1960 parecía haber quedado únicamente en los libros de historia, debido a la asociación generalizada con el Tercer Reich, pero la década de los 60 trajo consigo debates en torno a la autonomía reproductiva y al control natal. La década de los 70 vivió el debate sobre el aborto, las posturas provida y proelección, y fue así como poco a poco el tema de la eugenesia encontró nuevamente cabida a partir de nuevas tecnologías, como la fertilización in vitro, las diferentes técnicas en reproducción asistida, la amniocintésis, los bancos de semen, la ingeniería genética y, finalmente, la clonación. La gran diferencia sería que esta nueva eugenesia se diferenciaría de la anterior porque serían los individuos los que tendrían el poder de decisión.

\section{Eugenesia liberal}

La segunda mitad del siglo XX se caracterizó por el desarrollo acelerado de la biomedicina y la biología molecular, obtenido a partir del conocimiento del $\mathrm{ADN}$, que posibilitó la manipulación más efectiva e intencionada de nuestro genoma $y$, por ende, de la constitución hereditaria de la humanidad.

En la actualidad, la eugenesia positiva consiste en la aplicación del conocimiento biológico molecular, el diagnóstico y la intervención genética en la búsqueda del enriquecimiento de nuestro genotipo para modificar nuestro fenotipo, con la finalidad de obtener una descendencia que la selección natural probablemente nunca hubiera conseguido. Así, las nuevas tecnologías de manipulación genética permitirían realizar el deseo de los padres de no solo tener hijos saludables, sino también "virtuosos" y bien dotados, tanto física como intelectualmente(16). Pero, algo que preocupa a muchos, ¿qué debemos entender por estas "mejoras" genotípicas y fenotípicas? ¿Quién las define? ¿Qué valores y criterios guiarán esas intervenciones? ¿Cuál es el genotipo ideal? $(10,17,18)$.

Por otra parte, la "eugenesia negativa" busca corregir errores genéticos y eliminar enfermedades o factores genéticos desencadenantes de ellas. Las herramientas que pueden utilizarse son la ingeniería genética, la terapia génica germinal, el escaneo reproductivo, el diagnóstico genético preimplantatorio, cuando se trata de tratamientos de fertilización in vitro, y el diagnóstico prenatal, en caso de un embarazo intrauterino(19:676). De acuerdo con Daniel Sutullo, este tipo de eugenesia comprende decisiones individuales de tratamientos terapéuticos solo si su finalidad es influir sobre la herencia de características genéticas.

Recientemente, en un intento de rescatar el proyecto social del "bien nacer" y de realizar estudios prospectivos basados en las nuevas biotecnologías, surge la "eugenesia liberal", término acuñado por Nicholas Agar en 1999, caracterizada por tres aspectos diferenciales respecto de la eugenesia de Estado: 1) ser voluntaria, lo cual implica que la decisión de modificar la carga genética de los hijospor-nacer se efectúe sin coerción; 2) individual, esto es, realizada por núcleos familiares y únicamente con la finalidad de modificar su progenie, y no para alterar acervos genéticos de comunidades completas, y 3) independiente del Estado, para que este no promueva ningún aspecto genético, evitando así la creación de moldes $(20,21)$. De acuerdo con Michael Freeden(21), esta nueva propuesta permite, a cualquiera y por la razón que sea, ofrecer servicios genéticos a todos aquellos que los deseen y están dispuestos a aceptarlos en los términos en los que se ofrecen. En pocas palabras, la eugenesia liberal pretende extender las libertades reproductivas y procreativas de los futuros padres, con base en la disponibilidad de medios tecnológicos en el mercado.

El tipo de eugenesia al que se refiere Agar está fuertemente relacionada con la libertad reproductiva (reproductive choice en EE.UU.), que considera que una persona o pareja puede reproducirse con quien quiera, cuando quiera, por la razón que sea y cuantas veces lo desee. Junto con esta idea, la nueva eugenesia no considera que exista una línea divisoria entre las modificaciones ambientales y las genéticas, ya que en ambos casos el resultado es básicamente el mismo: mejorar a los futuros hijos. Sobre esta base, Agar introduce el concepto de "eugenesia liberal" (liberal eugenics).

Una de las características de la eugenesia liberal 
es que da primacía a lo que los individuos desean y la concepción de los que ellos consideran una buena vida, es decir, lo que para unos puede ser considerado una buena calidad de vida para otros puede ser simplemente inadmisible y esta valoración es netamente individual. El papel del Estado se restringe a facilitar, en lugar de imponer o restringir, las decisiones eugenésicas, permitiéndole a los padres elegir, conforme a sus nociones de lo que es la buena vida, mejoras eugenésicas que puedan proporcionarles a sus hijos(22).

La regulación de esta nueva eugenesia - $\mathrm{a}$ diferencia de su predecesora, que se encontraba en manos del gobierno- estaría en los propios mecanismos económicos del mercado, lo que generaría dos problemas evidentes: por un lado, la libertad de obtener los beneficios de estas nuevas tecnologías estaría al alcance de quienes pudieran pagarla y, por otro, el riesgo de que productos, objetivos y resultados de la investigación se vean corrompidos por intereses de compañías farmacéuticas transnacionales(23).

Queda claro que la eugenesia liberal presenta ventajas sobre la eugenesia totalitaria, así como desventajas. Del análisis comparativo entre ambas podemos determinar las características rescatables de cada una, con el fin de recuperar lo mejor de ambos modelos y basar en ellos la propuesta de una tercera vía eugenésica.

\section{Una tercera vía}

Del modelo totalitario podemos recuperar la importancia otorgada a la salud, a la educación en relación con la higiene, la importancia del medio ambiente, la alimentación, el cuidado maternoinfantil y las políticas de salud públicas, con la gran diferencia de que en esta ocasión será en beneficio de todos y no únicamente de aquellos considerados “mejores". Del modelo liberal podemos recuperar la autonomía — que sea individual y voluntaria-, dejando de lado la posibilidad de que sea solo el mercado el que, por medio de la oferta y la demanda, rija este nuevo modelo. El principal problema es que, de ser así, los únicos que podrán tener acceso a los nuevos tratamientos serán los que puedan pagarlos, exacerbando la diferencia entre ricos y pobres.

Considerando lo anterior, decidimos proponer un nuevo modelo de eugenesia. Uno que, en palabras de Sagols, sea una "interfaz" eugenésica entre dos facetas históricas del viejo ideal del mejoramiento de la condición humana $(24: 15)^{8}$. Nuestra hipótesis es que esta tercera vía será factible siempre y cuando cumpla con dos requisitos éticos fundamentales: a) que sea parte de un sistema social de salud que permita la redistribución de bienes y oportunidades de desarrollo, es decir, que sea una eugenesia redefinida en un marco de justicia distributiva; b) que se atenga a los principios de precaución y de responsabilidad en cuanto a las tecnologías que implemente.

Una tercera vía para la eugenesia estaría dirigida fundamentalmente a reducir enfermedades y discapacidades que producen desigualdades naturales en las sociedades, siempre y cuando los riesgos que impliquen las medidas eugenésicas sean racionalmente manejables y aceptables por la mayoría, y siempre y cuando los individuos tengan el poder de tomar la decisión final de emplear o no los medios eugenésicos sobre sí mismos y sobre su descendencia, cuando sea posible.

Actualmente estamos trabajando en el desarrollo de esta nueva propuesta en que la eugenesia se conciba no solo como una práctica médica invasiva, sino como una gama de prácticas que intervienen en la salud de las generaciones futuras en diferentes ámbitos.

Proponemos que sean medidas individuales en las cuales la decisión final dependerá de la pareja o mujer embarazada tomarlas o rechazarlas. Todas ellas están encaminadas a proporcionar un estado de salud básico a los niños y niñas que nacerán en los próximos años, con la esperanza de que ellos puedan elegir sus planes de vida sin tener obstáculos infranqueables por motivos de salud precaria o discapacidades.

\footnotetext{
${ }^{8}$ Eugenesia liberal y eugenesia de Estado, derechos individuales y bien social.
} 


\section{Referencias}

1. Clifford R. The eugenics movement. An encyclopedia. USA: Greenwood Press: 2005.

2. Miranda M, Vallejo G, (comps). Darwinismo social y eugenesia en el mundo latino. Argentina: Siglo XXI; 2005.

3. Müller-Hill B. Eugenics, the science and religion of the Nazis. En Caplan AL. When medicine went mad: Bioethics and the Holocaust. USA: Humana Press; 1992: 43-52,

4. Kevles D. In the name of eugenics. USA: Harvard University Press; 1985.

5. Saade M. ¿Quiénes deben procrear? Los médicos engenistas bajo el signo social. (México, 1931-1940). Cuicuilco ENAH México 2004; 11(31): 1-36.

6. Weikart R. Eugenics. En Mitcham C, (ed). Encyclopedia of Science, Technology, and Ethics. Vol. 2. USA: Thomson/Gale; 2005.

7. Dolan DV. Psychiatry, psychology, and human sterilization then and now: "therapeutic" or in the social interest? Ethical Human Psychology and Psychiatry 2007; 9(2): 99-108.

8. Leys N. Eugenics in Brazil. En Adams MB. The Wellborn Science, Eugenics in Germany, France, Brazil, and Russia. USA: Oxford University Press; 1990: 110-152.

9. Rohden F. Medicina, Estado y Reproducción en Brasil de inicios del siglo XX. Iconos 2007; 28(47-57).

10. Suárez L. Eugenesia y racismo en México. México: UNAM; 2005,

11. Urías Horcasitas B. Eugenesia y aborto en México (1920-1940). En Memoria Debate Feminista 2003; 27(14): 305-323.

12. Haas F. German Science and black racism: roots of the Nazi Holocaust. FASEB J 2008; 22: 332-337.

13. Berez TM, Weiss SF. The Nazi symbiosis: politics and human genetics at the Kaiser Wilhelm Institute. Endeavour 2004; 28(4): 172-177.

14. Fallwell L. Eugenics. En Shelton DL. Encyclopedia of Genocide and crimes against humanity, Tomo 1. USA: Thomson/Gale; 2005.

15. Buchanan A, Brock DW, Daniels N, Wilker D. Genética y Justicia. Madrid: Cambridge University Press; 2002.

16. Wasserman D. My fair baby: what's wrong with parents genetically enhancing treis children? En Gehring V, (ed). Genetic prospects: essays on biotechnology, ethics and public policy. Rowman \& Littlefield; 2003.

17. Kass L. Beyond Therapy: Biotechnology and the Pursuit of Happiness. Report of the President's Council on Bioethics, which was created by President George W. Bush on November 28, 2001, by means of Executive Order 13237.

18. Kass L. La sabiduría de la repugnancia. En Luna F, Riviera López E, (comps). Los desafíos éticos de la genética humana. México: UNAM, Instituto de Investigaciones Filosóficas: Fondo de Cultura Económica; 2005: 181-214.

19. Dekker M, (ed). Introduction of molecular genetics and genomics into clinical practice. Encyclopedia of diagnostic genomics and proteomics. USA; 2005: 676-680.

20. Agar N. Liberal eugenics. En Singer P, Kuhse H. Bioethics. An anthology. Blackwell Publishing; 1999.

21. Fox D. The Illiberity of "liberal eugenics". Ratio 2007; 20(1): 1-25.

22. Fenton E. Liberal eugenics and human nature: Against Habermas. Hasting Center Report 2006; 36(6): 3542.

23. O'Lery MM. Eugenesia actual: un nuevo desafío para las disciplinas metacientíficas. Estudios fronterizos, Universidad de Baja California 2006; 7(13): 29-42.

24. Sagols L. Interfaz bioética. México: UNAM- Fontamara; 2006.

Recibido: 7 de enero de 2011

Aceptado: 8 de marzo de 2011 